\title{
Structure and nanotribology of thermally deposited gold nanoparticles on graphite
}

\author{
Ebru Cihan ${ }^{a}$, Alper Özoğul ${ }^{b}$, Mehmet Z. Baykara ${ }^{a, b, *}$ \\ a UNAM - Institute of Materials Science and Nanotechnology, Bilkent University, Ankara 06800, Turkey \\ ${ }^{\mathrm{b}}$ Department of Mechanical Engineering, Bilkent University, Ankara 06800, Turkey
}

\section{A R T I C L E I N F O}

\section{Article history:}

Received 22 December 2014

Received in revised form 18 February 2015

Accepted 14 April 2015

Available online 24 April 2015

\section{Keywords:}

Atomic force microscopy

Friction force microscopy

Friction

Nanotribology

Nanoparticle

\begin{abstract}
A B S T R A C T
We present experiments involving the structural and frictional characterization of gold nanoparticles (AuNP) thermally deposited on highly oriented pyrolytic graphite (HOPG). The effect of thermal deposition amount, as well as post-deposition annealing on the morphology and distribution of gold on HOPG is studied via scanning electron microscopy (SEM) measurements, while transmission electron microscopy (TEM) is utilized to confirm the crystalline character of the nanoparticles. Lateral force measurements conducted via atomic force microscopy (AFM) under ambient conditions are employed to investigate the nanotribological properties of the gold nanoparticles as a function of normal load. Finally, the increase in lateral force experienced at the edges of the nanoparticles is studied as a function of normal load, as well as nanoparticle height. As a whole, our results constitute a comprehensive structural and frictional characterization of the AuNP/HOPG material system, forming the basis for nanotribology experiments involving the lateral manipulation of thermally deposited AuNPs on HOPG via AFM under ambient conditions.
\end{abstract}

(c) 2015 Elsevier B.V. All rights reserved.

\section{Introduction}

Despite the fact that friction is ubiquitous in nature, the fundamental physical principles that govern this interesting phenomenon are still not well understood. While the macroscopic laws of friction involving a linearly proportional relationship between the normal load $\left(F_{\mathrm{n}}\right)$ and the friction force $\left(F_{\mathrm{f}}\right)$ arising between two objects in contact have been well established since hundreds of years thanks to pioneering experiments by Amontons, Coulomb and others, the macroscopically observed proportionality constant $\mu$ (the so-called friction coefficient) cannot be derived from first principles as it constitutes a complex function of interface structure, chemistry and environmental factors including temperature and humidity [1]. Moreover, the unavoidable multi-asperity nature of interfaces formed by two macroscopic objects in contact [2] complicates the physical interpretation of macroscopic friction experiments, leading to substantial difficulties in the determination of the actual contact area $(A)$ between the two objects, among others.

To overcome the above-mentioned difficulties associated with macroscopic tribology (the science of friction, wear and lubrication)

\footnotetext{
* Corresponding author. Tel.: +90 3122903428.

E-mail address: mehmet.baykara@bilkent.edu.tr (M.Z. Baykara).
}

experiments, the research field of nanotribology has been introduced relatively soon after the invention of the atomic force microscope (AFM) [3,4]. The AFM, which can be thought of as a very high-resolution mechanical microscope, allows the recording of (sub-)nanometer-scale topography as well as normal and lateral forces experienced by a very sharp single asperity (radii of curvature usually on the order of $<10 \mathrm{~nm}$ ) in the form of a tip at the end of a micro-machined $\mathrm{Si} / \mathrm{SiO}_{2} / \mathrm{Si}_{3} \mathrm{~N}_{4}$ cantilever during the raster-scanning of a given sample surface under slight contact (normal forces on the order of a few to tens of $\mathrm{nN}$ ) [5]. Thanks to the single-asperity nature of the contact formed between the AFM tip and the sample surface, various nanotribology experiments conducted on a large number of sample surfaces over the last couple of decades have resulted in the precise determination of the effect of normal load, sliding velocity and temperature on frictional behavior at the nanoscale [6-8]. Moreover, phenomena such as stick-slip [9] and superlubricity [10] have been observed and largely explained, in many cases with substantial support from theory and computational work [11].

Among the material systems investigated nanotribologically using an AFM-based approach, nanoparticles on layered substrates such as highly oriented pyrolytic graphite (HOPG) [12,13] are of particular interest, primarily due to the fact that they readily present a heterogeneous sample surface where the effect of changing experimental parameters such as normal load on frictional 
behavior can be compared and contrasted on the nanoparticles themselves and the substrates. Moreover, there has been a recent increase in nanotribology experiments involving the deliberate lateral manipulation of nanoparticles on structurally well-defined substrates such as HOPG and the measurement of the associated frictional forces, as a model approach to study frictional effects in devices featuring sliding components on nano- and micrometer scales [14-19]. Consequently, the need for a comprehensive nanometer-scale characterization of the structural and frictional properties of such sample systems arises as a prerequisite for the correct interpretation of the above-mentioned nano-manipulation experiments, among others.

Motivated by the discussion above, we present in this contribution a comprehensive characterization of the structural and nanotribological properties of thermally deposited gold nanoparticles (AuNP) on HOPG substrates. The particular choice of this sample system is motivated by recently reported nanomanipulation experiments involving AuNPs under ultrahigh vacuum (UHV) conditions [18], as well as the fact that the growth mechanisms of AuNPs on HOPG have been studied via various approaches in the past [20-24]. In the following sections of this work, the effects of thermal deposition amount, as well as postdeposition annealing on nanoparticle morphology and distribution are discussed via scanning electron microscopy (SEM) measurements. Results reveal that a transformation in morphology from small, non-uniformly dispersed gold islands that are coalesced to form channeled thin films on HOPG to much larger, well-faceted, mostly hexagonal AuNPs with much lower substrate coverage takes place upon post-deposition annealing. Furthermore, highresolution transmission electron microscopy (TEM) images are utilized to confirm the crystalline character of the hexagon-shaped AuNPs. An extensive nanotribological analysis involving a number of AuNPs via AFM performed in ambient conditions allows the characterization of the friction force measured on the nanoparticles and the HOPG substrate as a function of normal load. Finally, an analysis of the increase in lateral force experienced at AuNP edges is presented as a function of normal load and nanoparticle height, revealing a linearly increasing trend for both parameters.

It is important to emphasize at this point that the results reported in this paper form a much-needed basis in terms of a comprehensive structural and tribological characterization for future nano-manipulation experiments to be conducted using the AuNP/HOPG material system under ambient conditions. In fact, it is observed that many AuNPs are frequently manipulated on the HOPG substrate during AFM measurements, opening the route towards the quantitative characterization of interfacial friction between crystalline surfaces. On the other hand, the present focus has been placed on immobile AuNPs stuck between HOPG steps and other structural features, suitable for a comprehensive structural and frictional characterization via AFM and other techniques.

\section{Experimental}

\subsection{Sample preparation}

HOPG substrates have been prepared by mechanical cleaving in air via adhesive tape and immediately transferred into the vacuum chamber of a thermal evaporation system (Vaksis PVD Vapor-3S). Thermal evaporation of 999.9 purity gold on HOPG substrates took place at a base pressure on the order of $5 \times 10^{-6}$ Torr and at a deposition rate of $0.1 \AA / s$ for total deposited amounts between $1 \AA$ and $40 \AA$. During deposition, the HOPG substrate was held at room temperature. After deposition, the gold-coated HOPG substrates were removed from the evaporation system for optional postdeposition annealing and further analysis via SEM, TEM and AFM.
Post-deposition annealing at temperatures ranging from $400{ }^{\circ} \mathrm{C}$ to $650^{\circ} \mathrm{C}$ and for annealing times on the order of $30 \mathrm{~min}$ to $4 \mathrm{~h}$ took place in a quartz tube furnace (Alser Teknik/ProTherm).

\subsection{Sample characterization via SEM and TEM}

Prior to structural and nanotribological characterization by AFM, samples prepared as detailed in the previous section have been analyzed via SEM (FEI Quanta 200 FEG, typically operated at $10 \mathrm{kV}$ ) to study the morphology and the distribution of AuNPs on HOPG. Additionally, high-resolution TEM (FEI Tecnai G2 F30, typically operated at $300 \mathrm{kV}$ ) has been utilized to confirm the crystalline structure of AuNPs via direct imaging as well as electron diffraction. The TEM samples have been prepared by mechanical cleavage of a thin layer of the gold-covered HOPG sample and subsequent sonication in ethanol, followed by drop-casting on a Cu grid (300 mesh).

\subsection{Nanotribological measurements performed via AFM}

Complementary to SEM and TEM measurements, AFM experiments have been utilized to characterize the structure as well as the nanotribological properties of the AuNP/HOPG sample system. A commercial AFM instrument (PSIA XE-100) has been operated under ambient conditions and in the contact mode to simultaneously measure the topography of the sample surface and the lateral forces arising between the tip and the sample during scanning. A single silicon cantilever (Nanosensors PPP-CONTR series, radius of curvature $\approx 10 \mathrm{~nm}$ ) has been used for all AFM measurements. To reliably determine the normal as well as the lateral forces detected during AFM measurements, the cantilever has been calibrated according to the methods reported by Sader et al. [25] and Varenberg et al. [26], respectively, resulting in a normal spring constant $k$ of $0.23 \mathrm{~N} / \mathrm{m}$ and a calibration constant $\alpha$ of $15.0 \mathrm{nN} / \mathrm{V}$ for the lateral force signal. While the details of both calibration techniques are described in the respective references, let us indicate here that the Sader et al. method allows the practical calculation of normal spring constants of AFM cantilevers based on a measurement of resonance frequency, quality factor and plan-view dimensions, taking into account the effect of the surrounding fluid medium (air) on cantilever oscillation via a hydrodynamic function [25]. On the other hand, the Varenberg et al. method, sometimes referred to as the improved wedge method, involves the measurement of the forward and backward lateral force signals on the sloped and flat faces of a commercial calibration grating with known feature sizes and dimensions, effectively allowing the calculation of lateral force calibration constants using force equilibrium arguments in conjunction with the determination of friction loop width and offset values at fixed normal loads [26]. In accordance with literature, the reported friction forces in our experiments have been calculated by considering the half-width of the friction loops formed by the recording of lateral forces during forward and backward scans [27].

\section{Results and discussion}

\subsection{Effect of deposition amount and post-deposition annealing on morphology}

In order to obtain a heterogeneous sample system suitable for nanotribological investigation via AFM consisting of individual AuNPs of defined shape and reasonable lateral separation on HOPG, the first preparation step involved the thermal evaporation of gold onto freshly cleaved HOPG substrates. The growth kinetics and morphological characteristics of thin films of gold on HOPG have received particular attention in the past, where typically nonuniform surface coverages have been observed due to the relatively low surface energy exhibited by the HOPG substrate [20-24]. While 

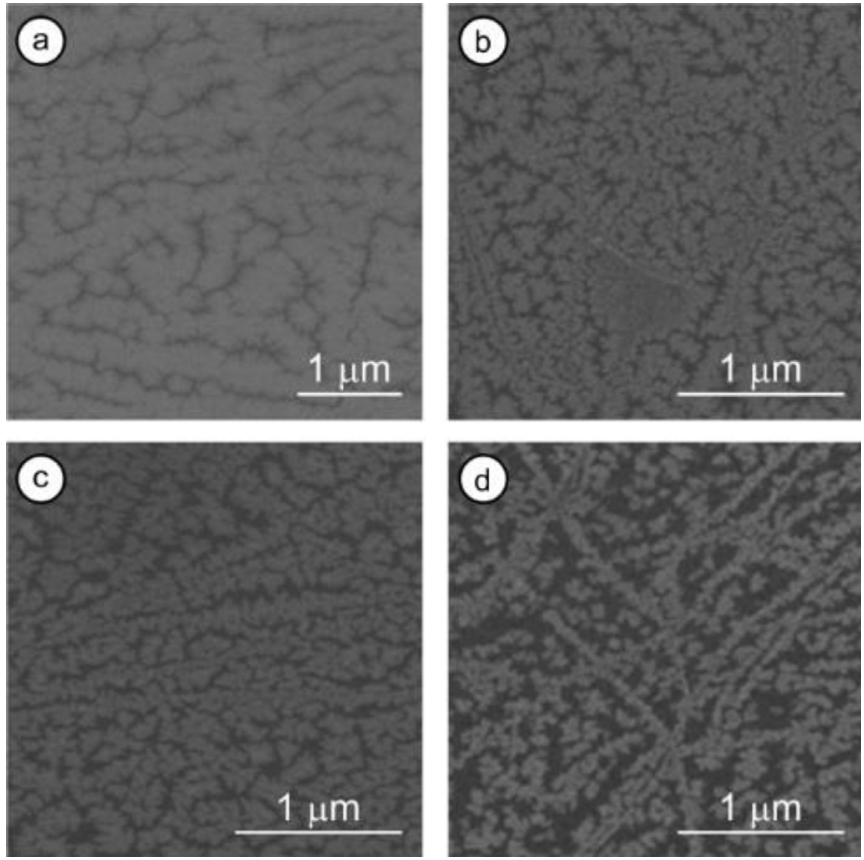

Fig. 1. SEM images of as-deposited Au thin films on HOPG for total deposition amounts of $20 \AA$ (a), $10 \AA$ (b), $5 \AA$ (c) and $1 \AA$ (d). Thermal evaporation has been performed with the HOPG substrate at room temperature, at a rate of $0.1 \AA / s$.

faceted and mostly hexagonal or triangular-shaped Au nanoparticles are obtained when evaporation is performed at elevated substrate temperatures, deposited films consisting of interconnected, elongated islands leading to a channeled morphology are expected for depositions where the substrate is held at room temperature.

Fig. 1 demonstrates the effect of thermal deposition amount on the resulting thin film morphology for film thicknesses of $20 \AA, 10 \AA, 5 \AA$ and $1 \AA$ via SEM images recorded post-deposition. Starting from a thickness of $20 \AA$, close to full surface coverage is observed; while at increasingly smaller film thicknesses, thin film morphologies comprising inter-connected, non-uniformly dispersed and irregularly-shaped gold islands are visible. The surface coverage and average island size gradually drop with smaller deposition amounts, until uncovered regions of the substrate on the order of several hundreds of $\mathrm{nm}$ become observable at a deposition amount of $1 \AA$. The fact that an accumulation of gold islands along certain linear features on the substrates becomes detectable at small deposition amounts such as 1 Å supports the argument that nucleation and growth primarily occurs at surface defects such as step edges and grain boundaries, in accordance with results from the literature [24].

While the SEM study presented in Fig. 1 regarding the morphology of gold thin films on HOPG as a function of deposition amount provides useful information, the resulting material system is unsuitable for precise nanotribological investigation via AFM due to the lack of structurally well-defined (faceted), large (hundreds of nm across) AuNPs at reasonable separations (at least several hundreds of $\mathrm{nm}$ ) from each other. In order to transform the as-deposited gold films into faceted AuNPs, post-deposition annealing in a quartz furnace has been performed at temperatures ranging from $400^{\circ} \mathrm{C}$ to $650^{\circ} \mathrm{C}$ and for annealing times on the order of $30 \mathrm{~min}$ to $4 \mathrm{~h}$. Please note that due to the relatively low surface coverage, samples comprising 1 Å of deposited gold on HOPG (Fig. 1(d)) have been employed for annealing. The results indicate that post-deposition annealing has a drastic effect on the morphology and distribution of gold on HOPG, leading to a severe
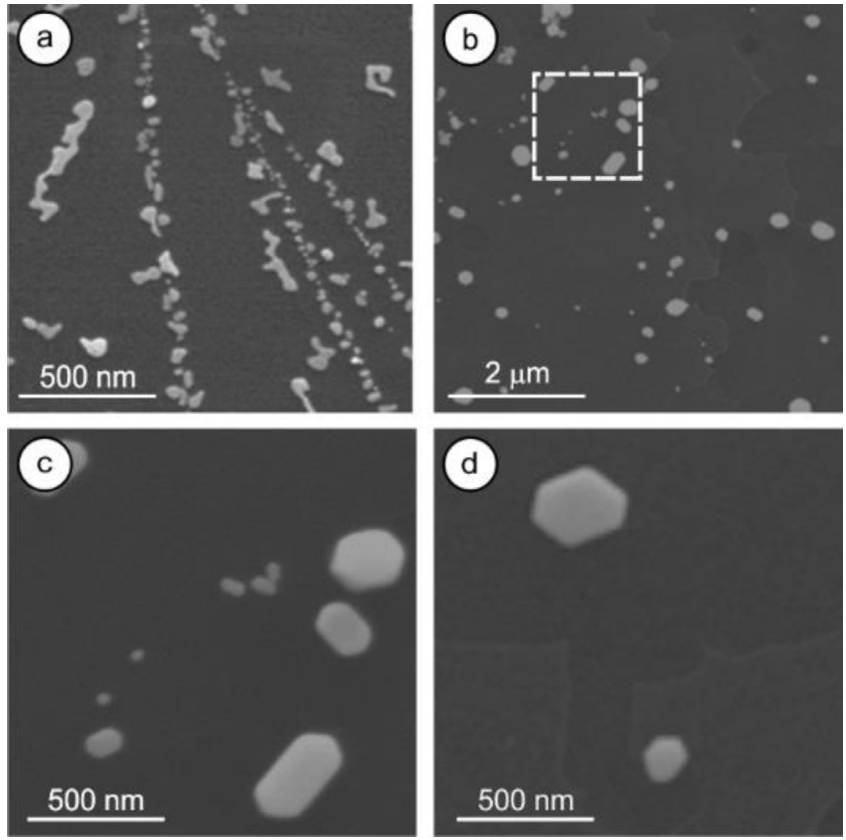

Fig. 2. SEM images of the AuNP/HOPG sample system after post-deposition annealing at (a) $500^{\circ} \mathrm{C}$ for $30 \mathrm{~min}$, (b, c) $600^{\circ} \mathrm{C}$ for $2 \mathrm{~h}$ and (d) $650^{\circ} \mathrm{C}$ for $2 \mathrm{~h}$. The SEM image presented in (c) is a zoomed-in view of the region of the sample surface designated by the dashed white rectangle in (b). The formation of welldefined, hexagonal/elongated-hexagonal AuNPs at elevated temperatures is readily observed.

reduction of surface coverage and significant coalescence. While annealing temperatures up to $500^{\circ} \mathrm{C}$ only result in the observation of non-faceted gold islands in elongated shapes (Fig. 2(a)) up to $4 \mathrm{~h}$ of annealing time; well-faceted, hexagonal/elongated-hexagonal AuNPs of $50-180 \mathrm{~nm}$ height and up to $500 \mathrm{~nm}$ in lateral dimensions are obtained at annealing temperatures of $600-650^{\circ} \mathrm{C}$ (Fig. $2(\mathrm{~b}-\mathrm{d})$ ), in accordance with recent studies performed on gold thin films on multilayer graphene [28]. Annealing at higher temperatures $\left(700^{\circ} \mathrm{C}\right.$ and above) has been observed to result in minor but detectable morphological damage on the HOPG surface and is thus prevented.

Due to the fact that thin films and clusters of metals deposited on various substrates (including, but not limited to metal oxides and layered materials such as mica and HOPG) present opportunities for devices with electronic, optical and magnetic properties that can be fine-tuned on the nanometer scale, the growth kinetics of such systems have been studied as a function of film thickness and annealing temperature in various studies in the past [29-32]. In particular, the growth of Au films on $\mathrm{Si}\left(\begin{array}{lll}1 & 1 & 1\end{array}\right)$ could be described by the dynamic scaling theory of growing interfaces, involving four stages that can be summarized as nucleation, lateral growth, coalescence and vertical growth; leading to an Avrami-type relationship between surface coverage and film thickness, which is characterized by rapid lateral growth at low coverages, followed by a damped coverage rate with increasing film thickness [29]. On the other hand, several careful post-deposition annealing experiments of $\mathrm{Au}$ on different substrates were utilized to interpret cluster formation within the framework of standard ripening models and resulted in the determination of surface diffusion coefficients and grain/cluster size distributions [29-32].

While the focus of our current work is on a comprehensive structural and nanotribological characterization of well-faceted, hexagonal AuNPs on HOPG and not on a detailed investigation of growth kinetics associated with this material system, we have nevertheless investigated the coverage of the HOPG substrate by Au as a function of film thickness (Fig. 3(a)), as well as the lateral size distribution of mostly-hexagonal AuNPs obtained after post-deposition 

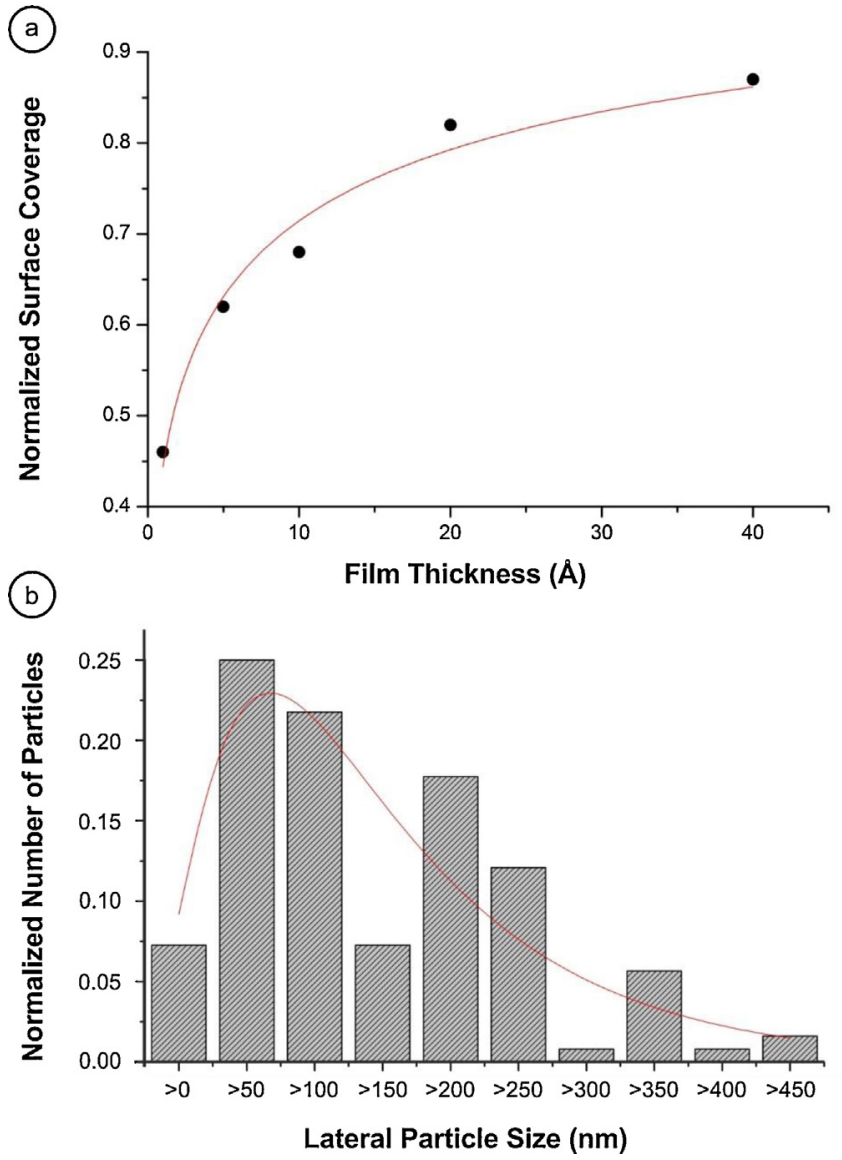

Fig. 3. (a) Surface coverage of Au on HOPG as a function of film thickness. An Avramitype fit represented by the red solid curve reasonably describes film growth [29]. (b) AuNP size distribution after post-deposition annealing at $600-650^{\circ} \mathrm{C}$. Lateral particle sizes up to $500 \mathrm{~nm}$ are observed. The solid red curve is a fit by the log-normal function [29].

annealing (Fig. 3(b)). Our results regarding surface coverage as a function of film thickness are in line with the previously mentioned dynamic scaling theory of growing interfaces, favoring a lateral growth and surface-diffusion-driven coalescence process, owing to the relatively weak chemical interactions between the adsorbed Au atoms and the HOPG surface (Fig. 3(a)) [29]. Moreover, we observe a relatively wide distribution of AuNP sizes up to $500 \mathrm{~nm}$ (Fig. 3(b)), with a mean particle size of $168 \pm 106 \mathrm{~nm}$. The main difference between the post-deposition-annealed AuNPs in our experiments and those that have been obtained on different substrates is that the AuNPs in our experiments are mostly hexagonal, whereas AuNPs obtained on substrates such as mica, $\mathrm{Si}(111)$ and $\mathrm{SiO}_{2}$ are mostly spherical in nature [29-31]. Postdeposition annealing experiments of Au films on graphene samples of different layer numbers have also led to the observation of hexagonal/triangular AuNPs, validating the proposition that weak interactions between the adsorbed Au atoms and the substrate lead to the preferential growth of crystalline, faceted AuNPs [28]. Let us finally indicate that the wide distribution of AuNP lateral size is favorable for nanotribological investigations, as it leads to the opportunity of performing future nano-manipulation experiments where interfacial friction forces acting on AuNPs of different size shall be quantified.

To sum up, the experimental results presented in this section constitute an alternative method for obtaining well-faceted AuNPs on HOPG substrates in the absence of the capability to increase substrate temperatures during thermal evaporation.
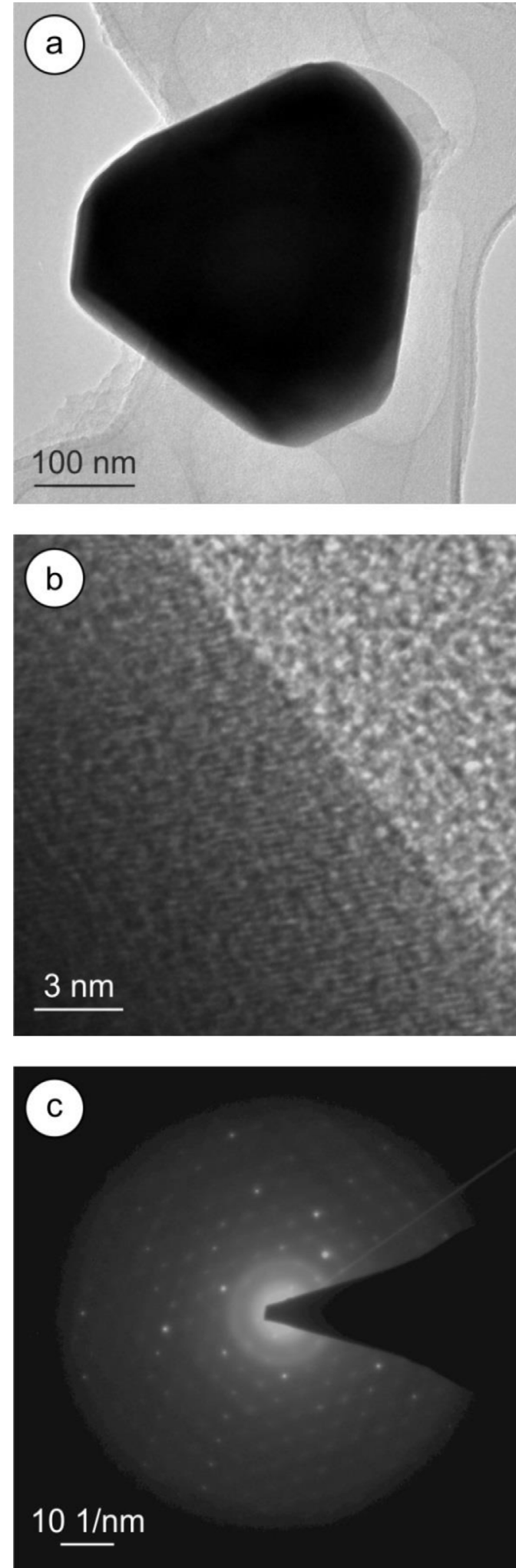

Fig. 4. TEM measurements performed on a single, well-faceted AuNP (a). While high-resolution imaging at the edge of the AuNP reveals crystalline order characterized by atomic stripes (b), diffraction data presented in (c) provides further confirmation for the crystallinity of the AuNP. 


\subsection{Confirmation of nanoparticle crystallinity via TEM}

It has been shown via a number of experiments and first principles calculations that friction occurring between two bodies in contact is a function of the physical properties of the interface - mainly its structure $[17,18,33]$. As such, in order to study carefully the effect of interface structure on friction at the nanoscale, structurally well-defined, i.e. crystalline, surfaces are a prerequisite. While the accurate determination of the atomicscale structure and chemistry associated with typical Si cantilever apices employed in AFM measurements remains a challenge, nanotribology experiments involving the lateral manipulation of nanoparticles via AFM have recently emerged as a viable approach where, e.g., the real contact area during sliding (the size of the nanoparticle-substrate interface) can be readily determined [19]. Pioneering nano-manipulation experiments conducted in this fashion primarily focused on Sb nanoparticles, which undergo a size-dependent phase transition from amorphous to crystalline at a particle size of $\sim 15,000 \mathrm{~nm}^{2}$ and are unavoidably covered by an amorphous antimony oxide shell when exposed to the ambient [17]. In contrast, AuNPs on substrates such as HOPG have very recently emerged as an alternative material system for nanomanipulation experiments, where the crystallinity of the interface should be conserved even under ambient conditions, allowing investigations regarding, e.g., structural lubricity to be performed under well-defined conditions [18].

Based on the idea that the results we present in this work should constitute a comprehensive structural and frictional characterization of the AuNP/HOPG material system, we have performed TEM measurement to confirm the crystalline character of thermally deposited and post-deposition annealed AuNPs on HOPG. The results of TEM investigations involving samples prepared as detailed in Section 2.2 are reported in Fig. 4. While the highresolution TEM image recorded at the edge of a hexagonal AuNP reveals the crystalline order of Au atoms (Fig. 4(a and b)), the associated characteristic diffraction pattern (Fig. 4(c)) provides further confirmation for the crystallinity of AuNPs. Based on the fact that the AuNPs investigated in the TEM study reported here have been exposed to ambient conditions for several weeks, the possibility for the formation of an oxide layer on AuNPs during relevant time frames for post-deposition AFM experiments can be readily excluded.

\subsection{Nanotribological properties of the AuNP/HOPG system investigated via AFM}

Despite the fact that the nanotribological properties of various materials investigated via AFM have been discussed widely in the literature, there are surprisingly few experimental reports on the nanotribology of metallic surfaces [34-37]. In addition to AFM experiments performed under ultrahigh vacuum (UHV) conditions on $\mathrm{Au}(1111)$ surfaces pointing towards a very low friction regime under small applied loads followed by a substantial increase in friction accompanied by wear [34], the capability to control the nanoscale frictional properties of the $\mathrm{Au}(111)$ surface immersed in an ionic liquid via the application of an electric potential has been demonstrated [37]. On the other hand, an extensive analysis of nanoscale frictional properties of gold nanoparticles thermally evaporated on HOPG has not been performed so far under ambient conditions. Considering that micro- and nano-scale functional devices featuring sliding components would be mostly used under ambient conditions for practical purposes, the need to perform a nanotribological study of related materials under such conditions-albeit at the expense of interface cleanliness-arises.

In order to perform a comparative study of the load dependence of nanoscale friction on AuNPs and HOPG substrates, individual
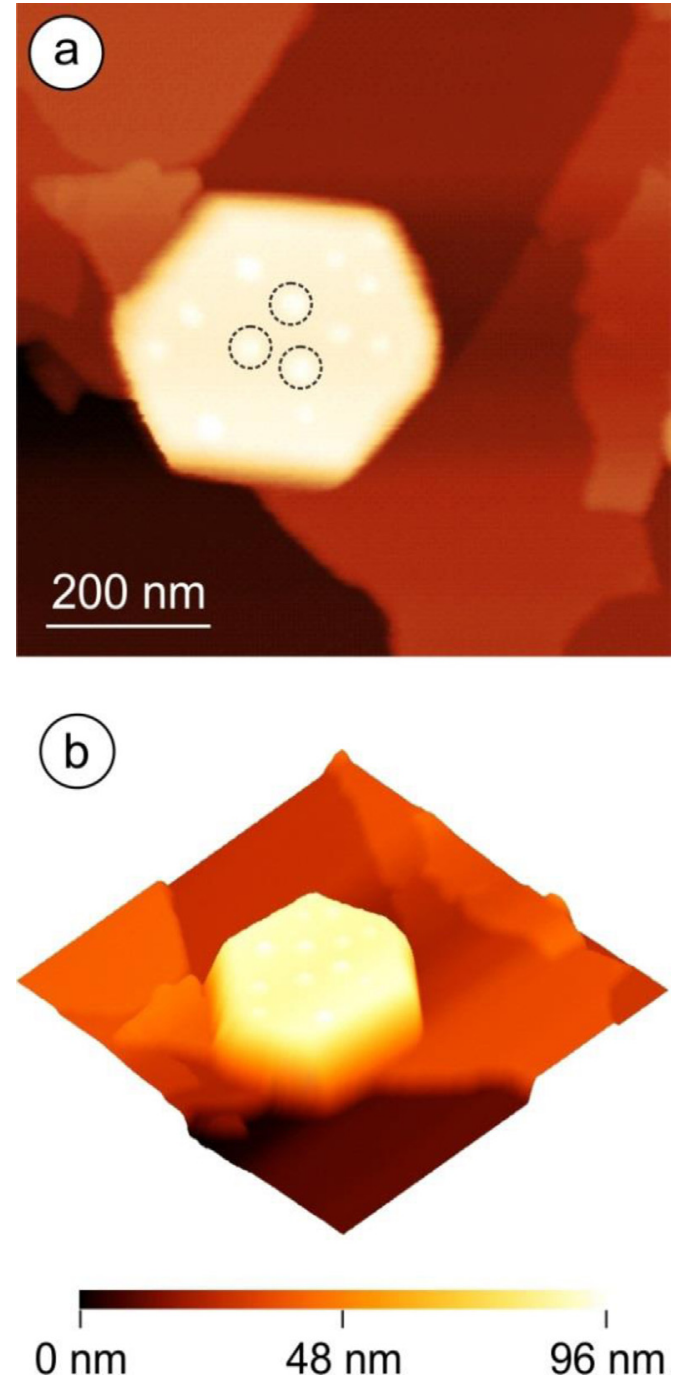

Fig. 5. Topographical map of a $\sim 75 \mathrm{~nm}$ high AuNP trapped between HOPG steps acquired via contact-mode AFM (a) and its 3D representation (b). Small mounds $(<10 \mathrm{~nm}$ in lateral dimensions) on top of the AuNP surface are highlighted.

nanoparticles have been imaged via contact mode AFM using the experimental methodology and equipment detailed in Section 2.3. Due to the fact that AuNPs on the HOPG substrates are frequently manipulated laterally during scanning based on the plowing action exhibited by the AFM tip, efforts have been directed at focusing on individual AuNPs trapped between the step edges of the HOPG substrate. In fact, many such nanoparticles can be observed during AFM experiments, presumably owing to the increased mobility exhibited by the coalescing gold clusters on the low-surface-energy HOPG substrate at elevated temperatures reached during annealing, resulting in lateral motion of AuNPs on the substrate and eventual trapping between step edges. Fig. 5 depicts a topography image associated with such a hexagon-shaped nanoparticle, together with a three-dimensional representation. While the specific nanoparticle depicted here exhibits a height of $\sim 75 \mathrm{~nm}$, AuNPs of 50-180 nm height have been observed during the experiments. Before switching to a discussion of lateral forces recorded on AuNPs, it should be indicated that topography images of certain AuNPs reveal the existence of small round mounds on the top surface (usually $\sim 10 \mathrm{~nm}$ across and $<10 \mathrm{~nm}$ in height), such as those highlighted in Fig. 5(a). While determining the exact nature of these features is beyond the scope of our work, it is predicted that the underlying 

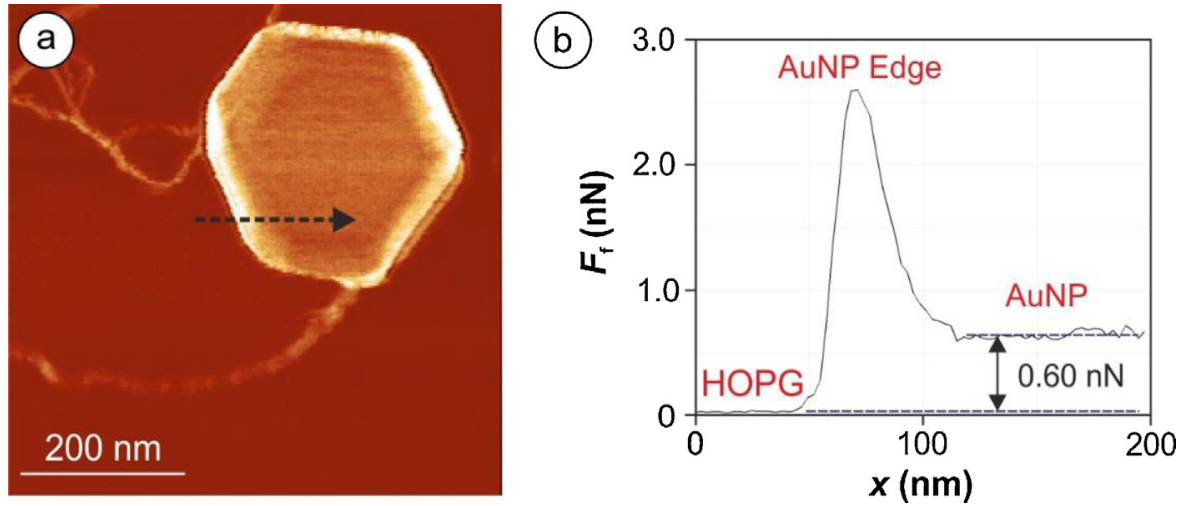

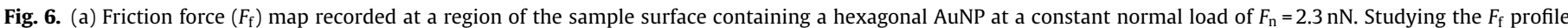

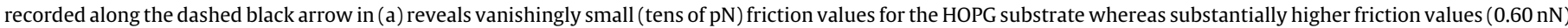
are observed on the AuNP (b). Please note the increased lateral forces experienced by the cantilever due to additional twisting at the edge of the $\sim 50$ nm high particle.

mechanism is related to the coalescence and growth characteristics exhibited by the AuNPs during post-deposition annealing.

To investigate the nanotribological properties of the Au/HOPG sample system, lateral forces acting between the tip and the sample surface during contact mode imaging have been recorded according to established procedures in the literature as indicated in Section 2.3. A typical lateral force map recorded on a region of the sample surface containing a nanoparticle is presented in Fig. 6(a). A study of the calibrated lateral force signal along the dashed section presented in Fig. 6(b) demonstrates a substantial difference in friction force on the order of $0.60 \mathrm{nN}$ measured on top of the AuNP as compared to the HOPG substrate, which exhibits vanishing friction. As expected, the lateral force value detected at the edge of the AuNP exhibits a sudden increase $(\sim 2.5 \mathrm{nN})$ due to the height-differenceinduced additional twisting of the cantilever [38].

The dependence of the friction force on the normal load at nanoscale contacts involving a single asperity (such as the AFM tip) on various substrates has been a topic of intense research since the establishment of the nanotribology field, mostly due to the main argument that understanding the frictional behavior of extended macro-scale, multi-asperity contacts could be enabled by the proper understanding of the physical mechanisms responsible for friction at single asperities. While several AFM experiments have revealed a specific " $2 / 3$ " power law dependence $\left(F_{\mathrm{f}} \sim F_{\mathrm{n}}{ }^{2 / 3}\right)$, arising from the assumption of a constant shear stress $\left(\tau=F_{\mathrm{f}} / A\right)$ within a Hertzian contact mechanics approach [39-43], there have been reports of linear dependence on certain sample surfaces, as well [44]. In order to further contribute to the experimental investigation of friction mechanisms at nanoscale asperities and to specifically characterize the nanotribological behavior of AuNPs in ambient conditions, the friction forces detected on 20 separate AuNPs have been studied as a function of normal load in our experiments (Fig. 7). Results reveal that the load dependence of friction forces on all AuNPs investigated under ambient conditions follows a " $2 / 3$ " power law within the error bars associated with our measurements, verifying the assumption of a constant shear stress and a Hertz-type deformation model for this sample system. An important point to indicate is the existence of a non-zero $F_{\mathrm{f}}(0.37 \mathrm{nN} \pm 0.11 \mathrm{nN})$ at zero normal load as a result of the finite adhesion force acting between the tip and AuNP surfaces under ambient conditions due to the existence of a water meniscus at the tip-sample contact [44]. Consequently, $F_{\mathrm{f}}$ becomes zero only at an effectively negative normal load $(-1.80 \mathrm{nN})$, corresponding to the pull-off force that needs to be applied to the cantilever to overcome adhesion and separate it from the sample surface. Let us note that force-distance spectroscopy experiments performed on
AuNPs on separate days have resulted in pull-off forces of magnitude $2.80 \pm 1.30 \mathrm{nN}$, which are in-line with our estimated value of $1.80 \mathrm{nN}$. It should be emphasized that the observation of a " $2 / 3$ " power law stemming from Hertzian contact mechanics in an AFM experiment requires the tip apex to closely resemble a spherical geometry (i.e., single-asperity) [42]. On the other hand, tip apices of multi-asperity character are expected to result in a linear relationship between $F_{\mathrm{f}}$ and $F_{\mathrm{n}}$, especially at $F_{\mathrm{n}}$ values several orders of magnitude higher than $1 \mathrm{nN}$ [42]. Based on the fact that our data validates a "2/3" power law relationship between $F_{\mathrm{f}}$ and $F_{\mathrm{n}}$ and has been acquired at loads $<25 \mathrm{nN}$, it is concluded that the specific tip apex geometry in our experiments can be considered to be of spherical, single-asperity character.

The absence of an ultralow friction regime and an abrupt increase in friction accompanied by substantial wear observed on Au surfaces under UHV conditions [34] is of particular scientific

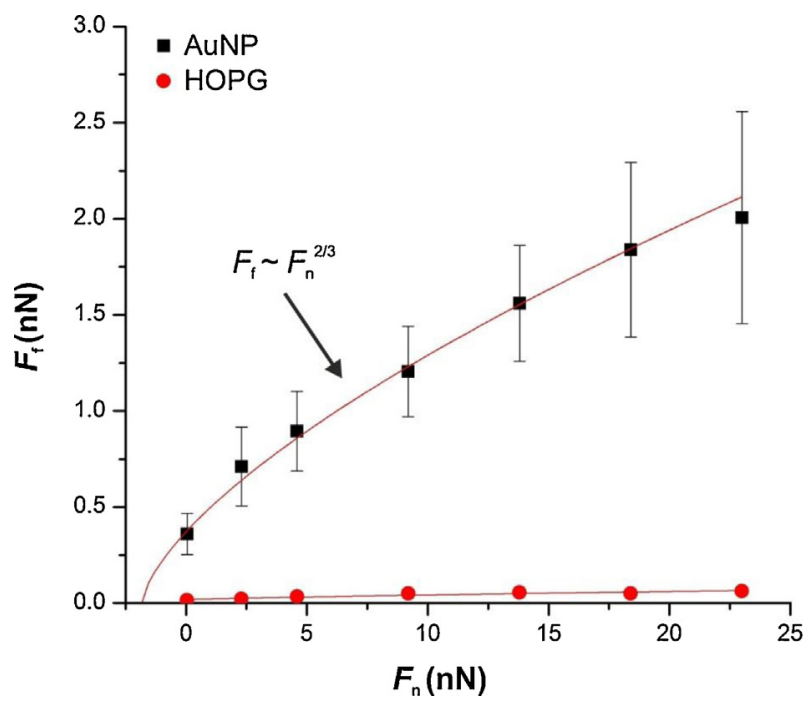

Fig. 7. Experimental data demonstrating the dependence of friction force $\left(F_{\mathrm{f}}\right)$ on normal load $\left(F_{\mathrm{n}}\right)$ for AuNPs as well as the HOPG substrate. While the recorded $F_{\mathrm{f}}$ values for the HOPG substrate are vanishingly small, AuNPs exhibit comparatively significant friction forces, which evolve according to a " $2 / 3$ " power law with increasing normal load, corresponding to the assumption of a constant shear stress and the validity of Hertzian contact. Error bars for the $F_{\mathrm{f}}$ values on AuNPs are calculated based on measurements performed on 20 separate nanoparticles with the same cantilever on separate days. Please note the pull-off force of $-1.80 \mathrm{nN}$, representing the effect of adhesion between the tip apex and the AuNPs. 


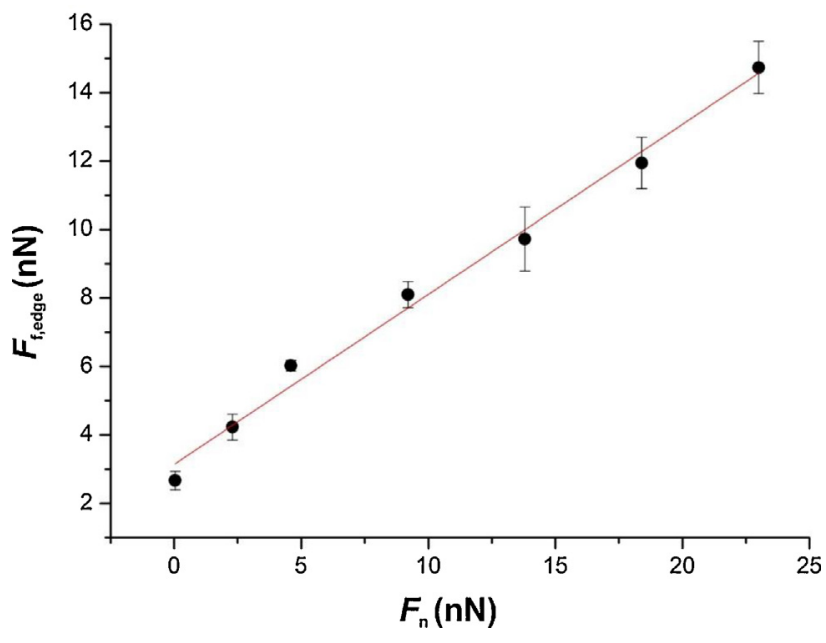

Fig. 8. Experimental data demonstrating the dependence of increased friction forces at AuNP edges $\left(F_{\mathrm{f} \text {,edge }}\right)$ on normal load $\left(F_{\mathrm{n}}\right)$ for a representative AuNP of $128 \mathrm{~nm}$ height. As expected from analytical calculations regarding the ratchet effect occurring during AFM measurements on sloped surfaces [38], $F_{f, \text { edge }}$ increases substantially with increasing $F_{\mathrm{n}}$, following a reasonably linear trend.

interest. In the mentioned experiments, these peculiar characteristics regarding friction on $\mathrm{Au}$ surfaces have been explained by the formation of a neck between the AFM tip and the clean gold surface due to the significant mobility exhibited by Au atoms at room temperature, and associated plastic deformations [35]. As the most probable mechanism for the lack of similar observations in our experiments performed in ambient conditions, the existence of a contamination layer adsorbed on the AuNP surfaces is of importance, an argument which is further supported by nanoscale friction experiments performed on Au surfaces modified with, e.g., self-assembled monolayers of alkanethiols which are devoid of effects associated with neck formation [45]. Finally, it can be readily inferred from Fig. 7 that the friction force values measured on HOPG are significantly lower than on AuNPs, owing to the outstanding lubrication properties of this layered material [39]. While a discussion of the fundamental principles associated with the exceptional lubrication properties of HOPG [46] is beyond the scope of this work, it should be indicated that the functional form of the dependence of $F_{\mathrm{f}}$ on $F_{\mathrm{n}}$ on HOPG is not straight-forward to determine due to the vanishingly small friction values measured [39].

It is well-known in the nanotribology community that topography-induced effects on the friction signal recorded via AFM are commonly encountered, especially on material systems that feature sudden changes in topography in the vertical direction. The AuNP/HOPG sample system investigated in our experiments presents an ideal opportunity to study related effects, as it comprises nanoparticles of $50-180 \mathrm{~nm}$ height situated on a substrate with wide, atomically flat terraces. The sharp increase in lateral forces encountered at, e.g., the edge of a AuNP as depicted in Fig. 6(b), can be attributed to the so-called ratchet effect, involving the partial contribution of applied normal load to the lateral force signal on a sloped surface, as well as the collision/impact undergone by the tip upon encountering a sloped surface, leading to additional twisting of the cantilever [38]. Utilizing the lateral force maps recorded on the AuNP/HOPG system in our experiments, we have performed a study of the increased lateral forces encountered at the edges of AuNPs $\left(F_{\mathrm{f} \text {,edge }}\right)$ as a function of normal load $F_{\mathrm{n}}$ and particle height $h$. Measurements performed on 20 separate AuNPs with different heights point towards a linearly proportional relationship between $F_{\mathrm{n}}$ and $F_{\mathrm{f} \text {,edge }}$ - the related data for a typical AuNP of $128 \mathrm{~nm}$ height is depicted in Fig. 8. Finally, Fig. 9 presents

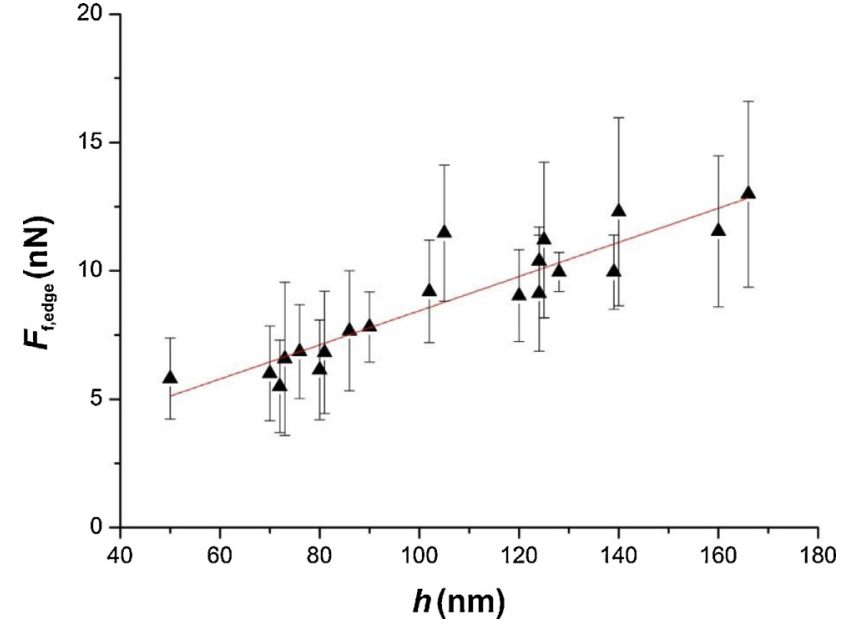

Fig. 9. Experimental data demonstrating the dependence of increased friction forces at AuNPedges $\left(F_{\mathrm{f}, \text { edge }}\right)$ on nanoparticle height $(h)$, acquired at a fixed normal load $F_{\mathrm{n}}$ of $13.8 \mathrm{nN}$. As it can be readily inferred from the data presented for 20 separate AuNPs with heights ranging from $50 \mathrm{~nm}$ to $166 \mathrm{~nm}, F_{\mathrm{f} \text {,edge }}$ displays a linearly proportional relationship with $h$. The error bars for $F_{\text {f,edge }}$ have been calculated by considering a large number of friction force profiles (such as the one presented in Fig. 6(b)) along the scanning direction of the cantilever for each AuNP at the given constant load.

an investigation towards the dependence of increased lateral forces detected at nanoparticle edges $F_{\mathrm{f} \text {,edge }}$ on nanoparticle height $h$. As one can infer from the experimentally obtained data, $F_{\mathrm{f} \text {,edge }}$ increases substantially with increasing $h$, following a linear trend within the error bars of our measurements.

\section{Conclusions}

We have presented the results of an experimental investigation aimed at a comprehensive structural and nanotribological characterization of thermally deposited gold nanoparticles on HOPG. Well-faceted, hexagonal/elongated-hexagonal AuNPs were formed upon post-deposition annealing in a quartz furnace at $600-650^{\circ} \mathrm{C}$, in strong contrast to as-deposited thin films exhibiting a channeled, irregular appearance with non-uniform coverage. The crystalline character of the nanoparticles was confirmed via high-resolution TEM imaging and diffraction measurements. To investigate the nanotribological properties of the nanoparticles as well as the substrate, AFM measurements performed via a single, calibrated cantilever have been utilized. In contrast to UHV measurements of nanoscale friction on gold surfaces, our measurements performed under ambient conditions point towards a " $2 / 3$ " power law dependence of friction on normal load for AuNPs, in accordance with the assumption of a constant shear stress during sliding and Hertzian contact mechanics in the presence of adhesion. Finally, a study of increased lateral forces at AuNP edges as a function of normal load and particle height has been performed, revealing a linearly proportional dependence for both parameters. Our experiments provide a comprehensive characterization of the structural and frictional properties of the AuNP/HOPG sample system, which emerges as an ideal candidate for future nano-manipulation experiments aimed at studying the fundamental mechanisms of friction under ambient conditions.

\section{Acknowledgements}

Financial support from the Marie Curie Actions of the European Commission's FP7 Program in the form of a Career Integration Grant (grant agreement no. PCIG12-GA-2012-333843), the Outstanding Young Scientist program of the Turkish Academy of Sciences (TÜBA-GEBIP) as well as the 2209/A program of TÜBITAK 
(The Scientific and Technological Research Council of Turkey) is gratefully acknowledged.

\section{References}

[1] B. Bhushan, Introduction to Tribology, Wiley, New York, 2013.

[2] J.A. Greenwood, J.B.P. Williamson, Contact of nominally flat surfaces, Proc. R. Soc. Lond. A 295 (1966) 300.

[3] B. Bhushan, J.N. Israelachvili, U. Landman, Nanotribology: friction, wear and lubrication at the atomic scale, Nature 374 (1995) 607.

[4] C.M. Mate, Tribology on the Small Scale: A Bottom Up Approach to Friction, Lubrication, and Wear, Oxford University Press, Oxford, 2008

[5] P.J. Eaton, P. West, Atomic Force Microscopy, Oxford University Press, Oxford, 2010.

[6] R.W. Carpick, M. Salmeron, Scratching the surface: fundamental investigations of tribology with atomic force microscopy, Chem. Rev. 97 (1997) 1163.

[7] I. Szlufarska, M. Chandross, R.W. Carpick, Recent advances in single-asperity nanotribology, J. Phys. D Appl. Phys. 41 (2008) 123001.

[8] H. Holscher, A. Schirmeisen, U.D. Schwarz, Principles of atomic friction: From sticking atoms to superlubric sliding, Philos. Trans. R. Soc. A 366 (2008) 1383.

[9] H. Holscher, U.D. Schwarz, O. Zworner, R. Wiesendanger, Consequences of the stick-slip movement for the scanning force microscopy imaging of graphite, Phys. Rev. B 57 (1998) 2477.

[10] M. Dienwiebel, G.S. Verhoeven, N. Pradeep, J.W.M. Frenken, J.A. Heimberg, H.W. Zandbergen, Superlubricity of graphite, Phys. Rev. Lett. 92 (2004) 126101.

[11] N.J. Mosey, M.H. Mueser, Atomistic Modeling of Friction, Rev. Comput. Chem. 25 (2007) 67.

[12] B. Stegemann, C. Ritter, B. Kaiser, K. Rademann, Crystallization of antimony nanoparticles: Pattern formation and fractal growth, J. Phys. Chem. B 108 (2004) 14292.

[13] N. Vandamme, E. Janssens, F. Vanhoutee, P. Lievens, C.V.C.M. Haesendonck, Scanning probe microscopy investigation of gold clusters deposited on atomically flat substrates, J. Phys. Condens. Matter 15 (2003) S2983.

[14] P.E. Sheehan, C.M. Lieber, Nanotribology and nanofabrication of MoO3 structures by force microscopy, Science 272 (1996) 1158.

[15] C. Ritter, M. Heyde, B. Stegemann, K. Rademann, U.D. Schwarz, Contact-area dependence of frictional forces: moving adsorbed antimony nanoparticles, Phys. Rev. B 71 (2005) 085405.

[16] D. Dietzel, C. Ritter, T. Monninghoff, H. Fuchs, A. Schirmeisen, U.D. Schwarz, Frictional duality observed during nanoparticle sliding, Phys. Rev. Lett. 101 (2008) 125505.

[17] C. Ritter, et al., Nonuniform friction-area dependency for antimony oxide surfaces sliding on graphite, Phys. Rev. B 88 (2013) 045422

[18] D. Dietzel, M. Feldmann, U.D. Schwarz, H. Fuchs, A. Schirmeisen, Scaling laws of structural lubricity, Phys. Rev. Lett. 111 (2013) 235502.

[19] D. Dietzel, U.D. Schwarz, A. Schirmeisen, Nanotribological studies using nanoparticle manipulation: Principles and application to structural lubricity, Friction 2 (2014) 114.

[20] T.P. Darby, C.M. Wayman, Nucleation and growth of gold films on graphite: I. Effects of substrate condition and evaporation rate, J. Cryst. Growth 28 (1975) 41.

[21] C.M. Wayman, T.P. Darby, Nucleation and growth of gold films on graphite: II. The effect of substrate temperature, J. Cryst. Growth 28 (1975) 53.

[22] R. Anton, I. Schneidereit, In situ TEM investigations of dendritic growth of Au particles on HOPG, Phys. Rev. B 58 (1998) 13874.

[23] R. Anton, P. Kreutzer, In situ TEM evaluation of the growth kinetics of Au particles on highly oriented pyrolithic graphite at elevated temperatures, Phys. Rev. B 61 (2000) 16077.
[24] Q.M. Guo, P. Fallon, J.L. Yin, R.E. Palmer, N. Bampos, J.M. Sanders, Growth of densely packed gold nanoparticles on graphite using molecular templates, Adv. Mater. 15 (2003) 1084

[25] J.E. Sader, J.W.M. Chon, P. Mulvaney, Calibration of rectangular atomic force microscope cantilevers, Rev. Sci. Instrum. 70 (1999) 3967.

[26] M. Varenberg, I. Etsion, G. Halperin, An improved wedge calibration method for lateral force in atomic force microscopy, Rev. Sci. Instrum. 74 (2003) 3362

[27] U.D. Schwarz, P. Koster, R. Wiesendanger, Quantitative analysis of lateral force microscopy experiments, Rev. Sci. Instrum. 67 (1996) 2560.

[28] H. Zhou, et al., The transformation of a gold film on few-layer graphene to produce either hexagonal or triangular nanoparticles during annealing, Carbon 52 (2013) 379.

[29] F. Ruffino, M.G. Grimaldi, Atomic force microscopy study of the growth mechanisms of nanostructured sputtered Au film on Si(111): Evolution with film thickness and annealing time, J. Appl. Phys. 107 (2010) 104321.

[30] F. Ruffino, A. Canino, M.G. Grimaldi, F. Giannazzo, C. Bongiorno, F. Roccaforte, V. Raineri, Self-organization of gold nanoclusters on hexagonal $\mathrm{SiC}$ and $\mathrm{SiO} 2$ surfaces, J. Appl. Phys. 101 (2007) 064306.

[31] F. Ruffino, V. Torrisi, G. Marletta, M.G. Grimaldi, Atomic force microscopy inves tigation of the kinetic growth mechanisms of sputtered nanostructured Au film on mica: towards a nanoscale morphology control, Nanoscale Res. Lett. 6(2011) 112.

[32] I. Beszeda, E.G. Gontier-Moya, A.W. Imre, Surface Ostwald-Ripening and Evaporation of Gold Beaded Films on Sapphire, Appl. Phys. A 81 (2005) 673.

[33] Y.F. Mo, K.T. Turner, I. Szlufarska, Friction laws at the nanoscale, Nature 457 (2009) 1116

[34] N.N. Gosvami, T. Filleter, P. Egberts, R. Bennewitz, Microscopic friction studies on metal surfaces, Tribol. Lett. 39 (2010) 19.

[35] R. Bennewitz, F. Hausen, N.N. Gosvami, Nanotribology of clean and modified gold surfaces, J. Mater. Res. 28 (2013) 1279.

[36] Q.Y. Li, Y.L. Dong, A. Martini, R.W. Carpick, Atomic friction modulation on the reconstructed Au (111) surface, Tribol. Lett. 43 (2011) 369.

[37] J. Sweeney, et al., Control of Nanoscale Friction on Gold in an Ionic Liquid by a Potential-Dependent Ionic Lubricant Layer, Phys. Rev. Lett. 109 (2012) 155502.

[38] S. Sundararajan, B. Bhushan, Topography-induced contributions to friction forces measured using an atomic force/friction force microscope, J. Appl. Phys. 88 (2000) 4825.

[39] U.D. Schwarz, O. Zworner, P. Koster, R. Wiesendanger, Quantitative analysis of the frictional properties of solid materials at low loads. I. Carbon compounds, Phys. Rev. B 56 (1997) 6987

[40] U.D. Schwarz, O. Zworner, P. Koster, P. Wiesendanger, Quantitative analysis of the frictional properties of solid materials at low loads. II. Mica and germanium sulfide, Phys. Rev. B 56 (1997) 6997.

[41] M. Enachescu, et al., Atomic Force Microscopy Study of an Ideally Hard Contact: The Diamond(111)/Tungsten Carbide Interface, Phys. Rev. Lett. 81 (1998 1877.

[42] E. Meyer, R. Luthi, L. Howald, M. Bammerlin, M. Guggisberg, H.J. Guntherodt Site-specific friction force spectroscopy, J. Vac. Sci. Technol. B 14 (1996) 1285.

[43] C.A.J. Putman, M. Igarashi, R. Kaneko, Single-asperity friction in friction force microscopy: the composite-tip model, Appl. Phys. Lett. 66 (1995) 3221.

[44] U.D. Schwarz, W. Allers, G. Gensterblum, R. Wiesendanger, Low-load friction behavior of epitaxial C 60 monolayers under Hertzian contact, Phys. Rev. B 52 (1995) 14976

[45] N.N. Gosvami, P. Egberts, R. Bennewitz, Molecular order and disorder in the frictional response of alkanethiol self-assembled monolayers, J. Phys. Chem. A 115 (2011) 6942

[46] M.Z. Baykara, et al., Exploring atomic-scale lateral forces in the attractive regime: a case study on graphite (0001), Nanotechnology 23 (2012) 405703. 